\title{
Modelagem para o acoplamento do transporte de umidade, calor e cloreto de sódio em estruturas de concreto
}

\author{
A model for coupled moisture, heat and sodium chloride \\ transport in concrete structures
}

\section{Rhayssa Maryell Marra Ribas \\ Gerson Henrique dos Santos Viviane Okita}

\section{Resumo}

A

durabilidade dos materiais está diretamente ligada a sua capacidade de resistir aos agentes de deterioração. Nesse contexto, as estruturas de concreto podem estar sujeitas aos efeitos da umidade e do sal, reduzindo consideravelmente o seu tempo de vida útil. Os sais que entram pelos poros do concreto podem atacar sua armadura, bem como se concentrar em alguns pontos e cristalizar, gerando trincas e fissuras e reduzindo, deste modo, a sua resistência. Nesse sentido, este trabalho apresenta um modelo matemático para o transporte acoplado de calor, umidade e sal em meios porosos. As equações governantes foram discretizadas por meio do método dos volumes finitos resolvidas simultaneamente a partir do algoritmo MultiTriDiagonal Matrix Algorithm (MTDMA). A verificação do modelo foi realizada a partir da comparação de estudos de casos obtidos na literatura, mostrando uma boa concordância entre os resultados.

Palavras-chave: Transporte de sal. Meio poroso. Concreto.

${ }^{1}$ Rhayssa Maryell Marra Ribas 'Universidade Tecnológica Federal do

Paraná Ponta Grossa - PR - Brasil

${ }^{2}$ Gerson Henrique dos Santos 2Universidade Tecnológica Federal do Ponta Grossa - PR - Brasil

${ }^{3}$ Viviane Okita ${ }^{3}$ Universidade Tecnológica Federal do Paraná Ponta Grossa - PR - Brasil

Recebido em 14/01/20 Aceito em 08/04/21

\section{Abstract}

The durability of materials is directly connected with deterioration agents. Hence, concrete structures can be subject to the effects of salt and moisture, significantly reducing their useful life. The salts that enter the concrete pores can attack its reinforcement. They can also accumulate at certain points and crystallize, leading to cracks and fractures and consequently reducing the resistance. This paper presents a mathematical model for the coupled heat, moisture and salt transportation in pores. The main equations were discretized through the finite volume method, solved simultaneously by the MultiTriDiagonal Matrix Algorithm. The verification of the model was made by comparing with studies from the literature and demonstrated good agreement between the outcomes.

Keywords: Salt transport. Porous media. Concrete. 


\section{Introdução}

A durabilidade de estruturas de concreto está diretamente relacionada com a capacidade de resistir aos agentes de deterioração. Segundo Helene (1997), estes agentes podem ser agressivos como o gás carbônico $\left(\mathrm{CO}_{2}\right)$, ácidos, sulfatos, reações álcali-agregados e sais de cloro que geram íons cloreto $\left(\mathrm{Cl}^{-}\right)$em meio aquoso. Estes últimos são um dos maiores causadores de deterioração em construções civis, dado que reduzem seu tempo de vida útil e trazem prejuízos econômicos.

No início do século XX, o conhecimento sobre a durabilidade das construções era empírico e se contava apenas com a experiência adquirida pelo profissional responsável. Com o passar dos anos e o aumento da tecnologia, o desenvolvimento de modelos que descrevem o comportamento de gases e líquidos em meios porosos tornou possível a previsão da deterioração do concreto e de outros materiais (MEDEIROS; ANDRADE; HELENE, 2011). De acordo com Martins (2006), é possível obter ganhos econômicos e ambientais a partir de melhorias na eficiência de processos geradas a partir do desenvolvimento da modelagem do transporte de calor e massa em meios porosos.

A compreensão a respeito do transporte de substâncias em meios porosos tem grande importância em diversas áreas, como engenharia de processos químicos, engenharia ambiental, reservatórios naturais, engenharia mecânica e construção civil. Segundo Mehta e Monteiro (2008), libera-se na atmosfera aproximadamente uma tonelada de $\mathrm{CO}_{2}$ para cada tonelada de concreto produzido. Este fato tem conscientizado o meio técnico quanto à necessidade de racionar o uso de matérias primas bem como reduzir o seu desperdício (PEDROSO, 2009; DELLINGHAUSEN et al., 2009).

A redução do tempo de vida das estruturas de concreto é consequência das diversas condições às quais estão submetidas durante seu ciclo de vida, como mudanças climáticas e de temperatura, níveis inadequados de tensão mecânica e ataques químicos e biológicos que causam diferentes tipos de danos. Um dos grandes motivos dessa deterioração pode estar na difusão de íons $\mathrm{Cl}^{-}$para o interior do meio poroso e em sua cristalização. Segundo Kropp (1995), existem duas formas de que esses íons entrem em contato com a estrutura. A primeira forma ocorre quando as matérias primas utilizadas para a fabricação do concreto já estão contaminadas ou os íons estão presentes em aditivos. A outra maneira, que é mais comum, é a presença do cloreto no ambiente onde a estrutura está exposta.

De acordo com dados da Organização das Nações Unidas, o setor da construção civil utiliza cerca de $40 \%$ de toda a energia e $25 \%$ de toda a água consumidas no mundo (BENITE, 2011). Sendo assim, o estudo da degradação do concreto armado devido à transposição de sal e umidade e a tentativa de torná-lo mais durável é de suma importância para melhorar sua eficiência, aumentar seu tempo de vida e diminuir os impactos ambientais gerados pelo setor da construção.

Nesse contexto, outros estudos já foram publicados envolvendo o transporte de umidade e sal em meios porosos. Arends et al. (2014) e Sun et al. (2018) consideraram o impacto dos processos de imbibição e secagem no transporte de umidade e sal em concreto. Li, Li e Chen (2008) propuseram diferentes difusividades para ambos os processos em seu modelo para o transporte de umidade. Ababneh, Benboudjema e Xi (2003) mostraram que o transporte de umidade em concretos parcialmente saturados acelera a entrada de íons e que esse fato deve ser considerado na modelagem. Derluyn, Moonen e Carmeliet (2014) citaram em seu estudo que, durante o processo de imbibição, ocorre a penetração de sais dissolvidos nos poros do concreto e que, no processo de secagem, ocorre a cristalização dos sais, que se expandem causando um aumento da pressão interna que eventualmente pode danificar e fissurar o concreto.

Entre outros importantes trabalhos encontrados na literatura, pode-se citar o de Rizzon e Andrade (2007), no qual o cimento Portland branco foi utilizado na análise da resistência e suscetibilidade para a penetração de cloretos. Por sua vez, Gorninski e Kazmierczak (2008) propuseram a utilização da resina poliéster isoftálica e ortoftálica como aglomerante do concreto. Por fim, Rocha e Póvoas (2019) buscaram obter um método mais eficiente para detectar a corrosão no concreto utilizando a termografia infravermelha e o ensaio de ultrassom.

No intuito de contribuir com o estudo da deterioração de estruturas de concreto pela ação de íons $\mathrm{Cl}^{-}$, propõe-se neste trabalho um modelo para o transporte acoplado de umidade, calor e sal em materiais porosos. A diferença de outros modelos encontrados na literatura, este modelo inclui a equação do transporte de energia, além do transporte de massa (umidade e sal) como uma das equações governantes. A discretização é realizada mediante o método dos volumes finitos (PATANKAR, 1980) e resolvidas simultaneamente a partir do algoritmo MTDMA (MENDES; PHILIPPI; LAMBERTS, 2002), o qual assegura a robustez numérica. Verificações realizadas por meio de outros estudos de casos encontrados na

144 Ribas, R. M. M.; Santos, G. H. dos; Okita, V. 
literatura mostram que o modelo apresentado se mostra promissor na análise da deterioração de estruturas de concreto, podendo promover melhorias tecnológicas que diminuam a necessidade de reparos precoces em novas construções, reduzindo, assim, os gastos e os impactos ambientais gerados pelo setor civil.

\section{Modelagem matemática}

Neste estudo, embora o termo de cristalização não tenha sido considerado nas equações de transporte, o modelo permite predizer quando os níveis críticos de saturação serão atingidos, iniciando o fenômeno. Adotou-se o critério de que os gradientes de concentração de sal não influenciam o transporte de umidade e calor no interior do meio poroso. Reconhece-se que a presença do sal pode trazer alguma influência nesse fluxo, no entanto, considerou-se que o transporte difusivo e convectivo do sal é influenciado pela pressão parcial de vapor, ocasionando um efeito muito mais significativo. A fixação do sal nas paredes dos poros também foi desprezada. Essas hipóteses também foram adotadas em outros trabalhos encontrados na literatura (ZANDEN; TAHER; ARENDS, 2015; ARENDS et al., 2014). Com relação às condições de contorno, diferentes combinações de fluxos e concentrações impostas de água e cloreto de sódio foram analisadas. Essas condições foram também utilizadas na maioria dos trabalhos descritos na seção anterior, com o objetivo de modelar os efeitos da névoa salina.

\section{Transporte de umidade}

Segundo Santos e Mendes (2009), o transporte de água no meio poroso pode ser dividido em dois fluxos, o de líquido e o de vapor (Equação 1):

$j=j_{l}+j_{v}$,

Eq. 1

Onde:

$j=$ fluxo de água $(\mathrm{kg} / \mathrm{m} \mathrm{s})$;

$j_{l}=$ fluxo de água no estado líquido $(\mathrm{kg} / \mathrm{m} \mathrm{s}) ; \mathrm{e}$

$j_{v}=$ fluxo de água no estado de vapor $(\mathrm{kg} / \mathrm{m} \mathrm{s})$.

O fluxo de líquido $\left(j_{l}\right)$ ocorre pelo efeito da gravidade e pelo transporte da fase condensada através da capilaridade, descrito pela equação de Darcy (Equação 2) adaptada para meios insaturados:

$j_{l}=K\left(\nabla P_{\text {suc }}-\rho_{l} g\right)$

Eq. 2

Onde (Equação 3):

$P_{\text {suc }}=P_{g}+P_{l}$

Eq. 3

Onde:

$K=$ condutividade hidráulica $(\mathrm{s})$;

$g=$ aceleração da gravidade $(\mathrm{m} / \mathrm{s})$;

$P_{s u c}=$ pressão de sucção $(\mathrm{Pa})$;

$\rho_{l}=$ densidade do líquido $[\mathrm{kg} / \mathrm{m}]$;

$\mathrm{P}_{\mathrm{g}}=$ pressão de gás $(\mathrm{Pa}) ; \mathrm{e}$

$\mathrm{P}_{1}=$ pressão de líquido $(\mathrm{Pa})$.

O gradiente da pressão de sucção pode ser descrito em função dos gradientes de temperatura e pressão parcial de vapor (Equação 4):

$\nabla P_{\text {suc }}=\frac{\partial P_{\text {suc }}}{\partial T} \nabla T+\frac{\partial P_{\text {suc }}}{\partial P_{v}} \nabla P_{v}$

Eq. 4

Onde (Equações 5 e 6):

$\frac{\partial P_{s u c}}{\partial T}=R_{v} \rho_{l}\left(-\ln \emptyset-\frac{T}{\emptyset}\left(-\frac{P_{v}}{P_{\text {sat }}^{2}} \frac{\partial P_{\text {sat }}}{\partial T}\right)\right)$

$\frac{\partial P_{s u c}}{\partial P_{v}}=-\frac{R_{v} T \rho_{l}}{P_{v}}$

Eq. 6

As Equações 5 e 6 foram derivadas a partir da Lei de Kelvin (Equação 7). 
$\ln (\varnothing)=\frac{-P_{s u c}}{R_{v} T \rho_{l}}$

Onde:

$\varnothing=$ umidade relativa;

$T=$ temperatura $[\mathrm{K}]$;

$P_{v}=$ pressão de vapor $[\mathrm{Pa}]$;

$P_{\text {sat }}=$ pressão de saturação [Pa]; e

$R_{v}=$ a constante dos gases para o vapor de água $[\mathrm{J} /(\mathrm{mol} . \mathrm{K})]$.

No caso do fluxo de vapor $\left(j_{v}\right)$, este ocorre de modo difusivo (Equação 8):

$j_{v}=-\delta_{v} \nabla P_{v}$

Onde: $\delta_{v}=$ permeabilidade difusiva ao vapor [s].

Deste modo, no modelo proposto, a Equação 9 descreve a conservação de umidade como:

$\frac{\partial w}{\partial t}=-\nabla \cdot j$

Onde: $w$ = conteúdo de umidade $(\mathrm{kg} / \mathrm{m})$.

Assim, para aumentar a robustez do método de solução (MTDMA), define-se o termo transiente em função da pressão parcial de vapor e da temperatura (Equação 10):

$\frac{\partial w}{\partial t}=\frac{\partial w}{\partial \emptyset} \frac{\partial \emptyset}{\partial P_{v}} \frac{\partial P_{v}}{\partial t}+\frac{\partial w}{\partial \emptyset} \frac{\partial \emptyset}{\partial T} \frac{\partial T}{\partial t}=\nabla \cdot\left[-K \frac{\partial P_{s u c}}{\partial T} \nabla T-\left(K \frac{\partial P_{s u c}}{\partial P_{v}}-\delta_{v}\right) \nabla P_{v}+K \rho_{l} g\right]$

Onde as derivadas (Equações 11 e 12):

$\frac{\partial \emptyset}{\partial P_{v}}=\frac{1}{P_{s a t}}$

$\frac{\partial \emptyset}{\partial T}=-\frac{P_{v}}{P_{\text {sat }}^{2}} \frac{\partial P_{\text {sat }}}{\partial T}$

Eq. 12

São obtidas a partir da definição de umidade relativa (Equação 13):

$\varnothing=\frac{P_{v}}{P_{\text {sat }}(T)}$

\section{Transporte de calor}

O fluxo de calor $q$, segundo Santos e Mendes (2009), pode ser atribuído aos efeitos da condução e da convecção (Equação 14):

$q=q_{\text {cond }}+q_{\text {conv }}$

Eq. 14

Em que (Equações 15 e 16):

$q_{\text {cond }}=-\lambda \nabla T$
$q_{\text {conv }}=\underbrace{j_{l} c_{p l} T}_{\begin{array}{c}\text { fluxo de } \\ \text { liquido }\end{array}}+\underbrace{j_{v} c_{p v} T}_{\begin{array}{c}\text { fluxo de } \\ \text { vapor }\end{array}}$

Eq. 15

Eq. 16

Onde:

$\lambda=$ condutividade térmica $(\mathrm{W} / \mathrm{mK})$;

$c_{p l}=$ calor específico da água líquida $(\mathrm{J} / \mathrm{kgK}) ; \mathrm{e}$

$c_{p v}=$ calor específico à pressão constante do vapor de água $(\mathrm{J} / \mathrm{kgK})$.

Deste modo, o balanço de energia é descrito pelo divergente do fluxo de calor mais um termo fonte, devido à transição de fase (Equação 17):

$c_{m} \rho_{0} \frac{\partial T}{\partial t}=-\nabla \cdot q+S$

Eq. 17

Em que (Equação 18):

146 Ribas, R. M. M.; Santos, G. H. dos; Okita, V. 
$c_{m}=c_{o}+\frac{c_{l} \theta}{\rho_{0}}$

Eq. 18

Onde:

$c_{0}=$ calor específico do material seco $(\mathrm{J} / \mathrm{kgK})$;

$c_{l}=$ calor específico da água líquida $(\mathrm{J} / \mathrm{kgK})$; e

$\rho_{0}=$ densidade do material seco $(\mathrm{kg} / \mathrm{m})$.

No caso do termo fonte, atribuída à mudança de fase, quantificou-se pelo calor latente de transição a partir do fluxo de vapor (Equação 19):

$S=-L(T) \nabla \cdot j_{v}$

Eq. 19

Onde: $L(T)=$ calor latente de vaporização $(\mathrm{J} / \mathrm{kg})$.

Desse modo, adotando-se a temperatura de referência de $0{ }^{\circ} \mathrm{C}$, descreve-se a equação da conservação da energia (Equação 20):

$c_{m} \rho_{0} \frac{\partial T}{\partial t}=\nabla \cdot\left(\left(\lambda-K \frac{\partial P_{s u c}}{\partial T} c_{p l} T\right) \nabla T-\left(K \frac{\partial P_{s u c}}{\partial P_{v}} c_{p l} T-\delta_{v} c_{p v} T\right) \nabla P_{v}+K \rho_{l} c_{p l} T g\right)-L(T) \nabla \cdot j_{v}$

\section{Transporte de sal}

Atribuiu-se o transporte de sal a dois fenômenos, conforme descrito por Arends et al. (2014). Por um lado, o transporte convectivo ocorre quando a água carrega o sal dissolvido através dos poros. Por outro lado, a difusão ocorre devido à diferença de concentração de sal entre os poros. Assim, o transporte de sal pode ser definido como descrito na Equação 21:

$\frac{\partial C_{S}}{\partial t}=\frac{\partial}{\partial x}\left(D \frac{\partial w}{\partial x} \frac{C_{S}}{w}+D_{s} \frac{\partial C_{S}}{\partial x}\right)$

Onde:

$C_{s}=$ concentração de sal $[\mathrm{kg} / \mathrm{m}]$;

$D=$ coeficiente de difusão da água no concreto $[\mathrm{m} / \mathrm{s}]$; e

$D_{s}=$ coeficiente de dispersão do sal no concreto [m /s], dado pela Equação 22 (ARENDS et al., 2014):

$D_{s}=\frac{D_{m}}{\tau}+\frac{1}{2}\left(\frac{D}{w} \frac{\partial w}{\partial x}\right)^{2} \frac{r^{2}}{D_{m}}$

Onde:

$D_{m}=$ coeficiente de difusão do sal na água $[\mathrm{m} / \mathrm{s}]$;

$\tau=$ tortuosidade do meio; e

$r=$ raio do poro $[\mathrm{m}]$.

\section{Discretização e algorítmo genérico implementado}

As discretizações das três equações governantes foram feitas aplicando o método dos volumes finitos abordado por Patankar (1980) em um domínio unidimensional, utilizando coordenadas cartesianas de modo totalmente implícito. A Figura 1 apresenta um volume de controle, em que a letra $\mathrm{P}$ representa o nó selecionado e as letras $\mathrm{W}$ e $\mathrm{E}$ representam os nós vizinhos a $\mathrm{P}$, todos localizados no centro de seus respectivos volumes de controle. Dessa forma, os coeficientes de transporte são calculados pela média harmônica na interface dos volumes de controle. 
Figura 1 - Volume de controle unidimensional

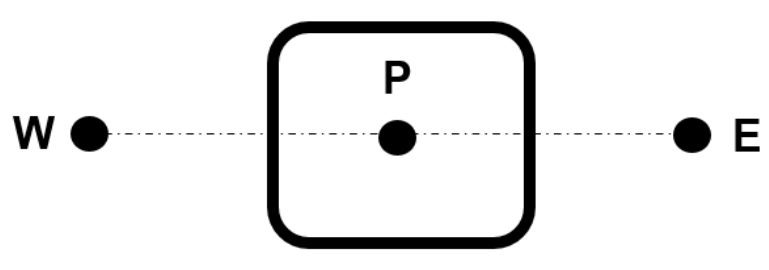

O resultado da discretização para a conservação de umidade, calor e sal para os volumes intermediários é apresentado nas Equações 23, 24 e 25, respectivamente. Os subíndices 0, 1 e 2 representam a qual equação o coeficiente pertence (umidade, sal ou calor, respectivamente).

$\left(\frac{C_{P v 0}}{\Delta t}+\frac{D_{P v e 0}}{\Delta x^{2}}+\frac{D_{P v w 0}}{\Delta x^{2}}\right) P_{v p}+\left(\frac{C_{T 0}}{\Delta t}+\frac{D_{T e}}{\Delta x^{2}}+\frac{D_{T w 0}}{\Delta x^{2}}\right) T_{p}=\frac{D_{T e} 0}{\Delta x^{2}} T_{e}+\frac{D_{P v e 0}}{\Delta x^{2}} P_{v e}+\frac{D_{T w 0}}{\Delta x^{2}} T_{w}+\frac{D_{P v w 0}}{\Delta x^{2}} P_{v w}+\frac{C_{p v 0}}{\Delta t} P_{v p}{ }^{0}+\frac{C_{T 0}}{\Delta t} T_{p}{ }^{0} \quad$ Eq. 23

$\left(\frac{D_{P v e 2}}{\Delta x^{2}}+\frac{D_{P v w 2}}{\Delta x^{2}}\right) P_{v p}+\left(\frac{C_{T 2}}{\Delta t}+\frac{D_{T e 2}}{\Delta x^{2}}+\frac{D_{T w 2}}{\Delta x^{2}}\right) T_{p}=\frac{D_{T e 2}}{\Delta x^{2}} T_{e}+\frac{D_{P v e 2}}{\Delta x^{2}} P_{v e}+\frac{D_{T w 2}}{\Delta x^{2}} T_{w}+\frac{D_{P v w 2}}{\Delta x^{2}} P_{v w}+\frac{C_{T 2}}{\Delta t} T_{p}{ }^{0} \quad$ Eq. 24

$\left(\frac{D_{P v e l}}{\Delta x^{2}}+\frac{D_{P w w 1}}{\Delta x^{2}}\right) P_{v p}+\left(\frac{D_{T e l}}{\Delta x^{2}}+\frac{D_{T w l}}{\Delta x^{2}}\right) T_{p}+\left(\frac{1}{\Delta t}+\frac{D_{C s e l}}{\Delta x^{2}}+\frac{D_{C s w 1}}{\Delta x^{2}}\right) C_{s p}=\frac{D_{T e l}}{\Delta x^{2}} T_{e}+\frac{D_{P v e l}}{\Delta x^{2}} P_{v e}+\frac{D_{C s e l}}{\Delta x^{2}} C_{s e}+\frac{D_{T w l}}{\Delta x^{2}} T_{w}+\frac{D_{P_{w w 1}}}{\Delta x^{2}} P_{v w}+\frac{D_{C s w 1}}{\Delta x^{2}} C_{s w}+\frac{C_{s}^{0}}{\Delta t} \quad$ Eq. 25

Onde:

$C_{P v 0}=\frac{\partial w}{\partial \emptyset} \frac{\partial \emptyset}{\partial P_{v}}$

$C_{T 0}=\frac{\partial w}{\partial \emptyset} \frac{\partial \emptyset}{\partial T}$

$D_{T 0}=K \frac{\partial P_{s u c}}{\partial T}$

$D_{P v 0}=K \frac{\partial P_{s u c}}{\partial P_{v}}+\delta_{v}$

$D_{T 1}=D \frac{C_{s}}{w}$

$D_{P v l}=\frac{\partial w}{\partial P v} \frac{D_{s}}{w}$

$D_{C s l}=D_{s}$

$C_{T 2}=c_{m} \rho_{0}$

$D_{T 2}=\lambda-K \frac{\partial P_{s u c}}{\partial T} c p_{l} T$

$D_{P v 2}=K \frac{\partial P_{s u c}}{\partial P v} c p_{l} T-\delta_{v} c p_{v} T$

Para solucionar o sistema de equações algébricas obtido pela discretização em volumes finitos foi utilizado o algoritmo MTDMA, proposto por Mendes, Philippi e Lamberts (2002), o qual permite a resolução simultânea do sistema de equações assegurando a robustez numérica.

A partir da discretização das equações governantes no domínio, são obtidas as equações na forma descrita pelas Equações 26, 27, 28, 29 e 30:

$A_{i} \cdot x_{i}=B_{i} \cdot x_{i+1}+C_{i} \cdot x_{i-1}+D_{i}$

Eq. 26

Portanto (Equação 27):

$$
A_{i}=\begin{array}{ccc}
\frac{C_{P v 0}}{\Delta t}+\frac{D_{P v e 0}}{\Delta x^{2}}+\frac{D_{P v w 0}}{\Delta x^{2}} & 0 & \frac{C_{T 0}}{\Delta t}+\frac{D_{T e}}{\Delta x^{2}}+\frac{D_{T w 0}}{\Delta x^{2}} \\
\frac{D_{P v e 2}}{\Delta x^{2}}+\frac{D_{P v w 2}}{\Delta x^{2}} & 0 & \frac{C_{T 2}}{\Delta t}+\frac{D_{T e 2}}{\Delta x^{2}}+\frac{D_{T w 2}}{\Delta x^{2}} \\
\frac{D_{P v e l}}{\Delta x^{2}}+\frac{D_{P v w 1}}{\Delta x^{2}} & \frac{1}{\Delta t}+\frac{D_{C s e l}}{\Delta x^{2}}+\frac{D_{C s w 1}}{\Delta x^{2}} & \frac{D_{T e l}}{\Delta x^{2}}+\frac{D_{T w 1}}{\Delta x^{2}}
\end{array}
$$

148 Ribas, R. M. M.; Santos, G. H. dos; Okita, V. 


$$
\begin{aligned}
& \frac{D_{P v e 0}}{\Delta x^{2}} \quad 0 \quad \frac{D_{T e \ell}}{\Delta x^{2}} \\
& B_{i}=\frac{D_{P v e l}}{\Delta x^{2}} \quad \frac{D_{C s e l}}{\Delta x^{2}} \quad \frac{D_{T e l}}{\Delta x^{2}} \\
& \frac{D_{P v e 2}}{\Delta x^{2}} \quad 0 \quad \frac{D_{T e 2}}{\Delta x^{2}} \\
& \frac{D_{P v w 0}}{\Delta x^{2}} \quad 0 \quad \frac{D_{T w 0}}{\Delta x^{2}} \\
& C i=\frac{D_{P v w 1}}{\Delta x^{2}} \quad \frac{D_{C s w 1}}{\Delta x^{2}} \quad \frac{D_{T w 1}}{\Delta x^{2}} \\
& \begin{array}{lll}
\frac{D_{P v w 2}}{\Delta x^{2}} & 0 & \frac{D_{T w 2}}{\Delta x^{2}}
\end{array} \\
& \frac{C_{p v 0}}{\Delta t} P_{v p}{ }^{0}+\frac{C_{T 0}}{\Delta t} T_{p}{ }^{0} \\
& D_{i}=\quad \frac{C_{s}^{0}}{\Delta t} \\
& \frac{C_{T 2}}{\Delta t} T_{p}{ }^{0}
\end{aligned}
$$

Onde: $\mathrm{x}_{\mathrm{i}}=$ vetor que contém as variáveis dependentes representadas pela Equação 31:

$x_{i}=\left[\begin{array}{c}w_{i} \\ C_{s} \\ T_{i}\end{array}\right]$

No método tradicional (TriDiagonal Matrix Algorithm [TDMA]), os coeficientes A, B, e C são tensores de primeira ordem. No MTDMA, que foi o método empregado para este trabalho, os tensores passaram a ser de segunda ordem. Neste caso, o vetor $\mathrm{x}_{i}$ pode ser expresso como uma função de $\mathrm{x}_{\mathrm{i}+1}$ (Equação 32):

$x_{i}=P_{i} \cdot x_{i+1}+q_{i}$

Eq. 32

Onde: $\mathrm{P}_{\mathrm{i}}$ e $\mathrm{q}_{\mathrm{i}}$ podem ser representados, respectivamente, pelas Equações 33 e 34:

$P_{i}=\left[\left(A_{i}-C_{i} \cdot P_{i-1}\right)^{-1} \cdot B_{i}\right]$

$q_{i}=\left(A_{i}-C_{i} \cdot P_{i-1}\right)^{-1}\left(C_{i} \cdot q_{i-1}+D_{i}\right)$

\section{Resultados e discussões}

Com o objetivo de verificar o modelo proposto, alguns testes foram realizados e comparados com os estudos de casos apresentados por Zanden, Taher e Arends (2015). As simulações foram feitas para uma parede de concreto inserida em um ambiente marítimo com névoa salina de íons $\mathrm{Cl}^{-}$. Considerou-se a superfície interna da parede como adiabática e impermeável, enquanto a superfície externa foi submetida ao ambiente externo. As condições iniciais e os parâmetros de simulação estão detalhados no Quadro 1.

Para representar a variação da umidade de acordo com as estações do ano, Zanden, Taher e Arends (2015) propuseram a Equação 35, onde a quantidade de água na amostra (w) muda de acordo com uma função seno ao longo do tempo $(\mathrm{t})$ :

$w=70 \operatorname{sen}\left(1,9924 \times 10^{-7} t\right)+80$

A Figura 2 mostra uma representação da variação da umidade da amostra de acordo com as estações do ano.

Neste estudo de caso, a temperatura do sistema foi considerada constante. Como descrito nas hipóteses do modelo, não há interferência do gradiente de sal no transporte de umidade. Dessa forma, a Figura 3 apresenta os perfis da quantidade de água na amostra para os quatro casos analisados na sequência.

Como pode-se observar na Figura 3, os perfis do conteúdo de água do modelo proposto e o apresentado por Zanden, Taher e Arends (2015) são bastante semelhantes. A figura também mostra que os perfis da quantidade de água variam em torno de $90 \%$ ao longo do ano entre a estação mais úmida e a mais seca, permitindo uma boa distinção entre as estações.

A Tabela 1 apresenta as condições de contorno dos casos aplicados na verificação do modelo proposto. 
Quadro 1 - Condições iniciais e parâmetros de simulação

\begin{tabular}{|l|l|}
\hline Comprimento da parede & $0,1 \mathrm{~m}$ \\
\hline Temperatura $(\mathbf{T})$ & $293,15 \mathrm{~K}$ \\
\hline Umidade inicial $(\mathbf{w})$ & $80 \mathrm{Kg} \mathrm{H}{ }_{2} \mathrm{O} / \mathrm{m}$ concreto \\
\hline Coeficiente de difusão do cloreto $\left(\mathbf{C l}^{-}\right)$em água $\left(\mathbf{D}_{\mathbf{m}}\right)$ & $1,26.10^{-9} \mathrm{~m} / \mathrm{s}$ \\
\hline Passo de tempo $(\Delta \mathbf{t})$ & $10800 \mathrm{~s}(3 \mathrm{horas})$ \\
\hline Quantidade de volumes & 20 \\
\hline
\end{tabular}

Figura 2 - Entrada de água na amostra de acordo com o tempo

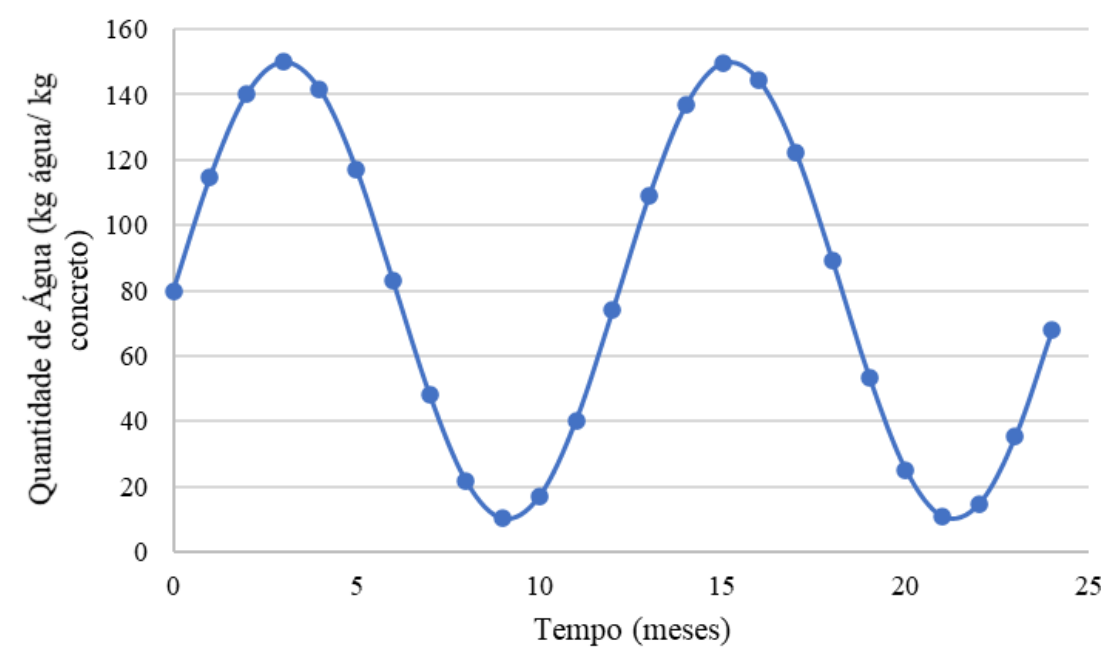

Figura 3 - Perfil do conteúdo de água ao longo da amostra

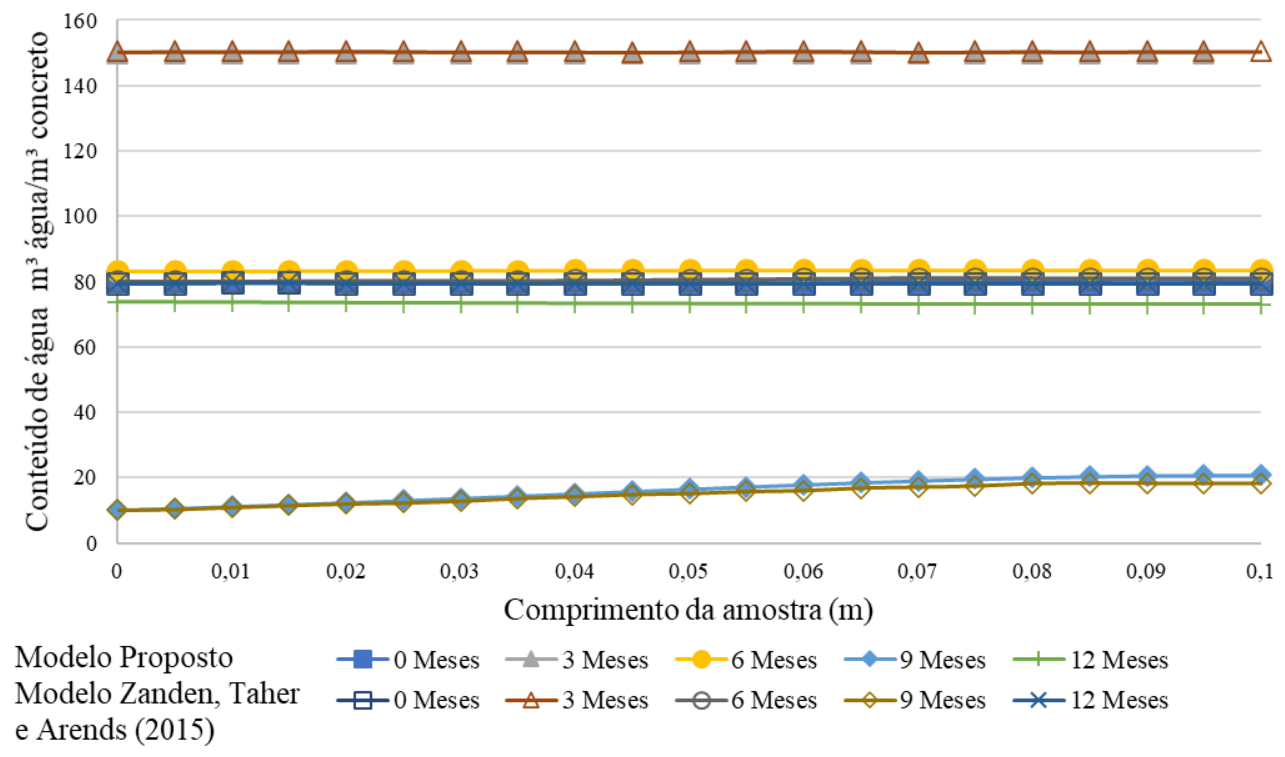

Tabela 1 - Casos simulados para a verificação do modelo proposto

\begin{tabular}{c|c|c|c|c}
\hline Caso & $\mathbf{1}$ & $\mathbf{2}$ & $\mathbf{3}$ & $\mathbf{4}$ \\
\hline Quantidade de sal inicial $\left(\mathbf{k g} / \mathbf{m}^{\mathbf{3}}\right)$ & 1 & 1 & 0 & 0 \\
\hline $\begin{array}{c}\text { Concentração da névoa salina } \\
\left(\mathbf{k g} / \mathbf{m}^{\mathbf{3}}\right)\end{array}$ & 0 & 0 & 19 & 19 \\
\hline Fluxo de entrada & Água & Água & Água e sal & Água e sal \\
\hline Fluxo de saída & Água & Água e sal & Água & Água e sal \\
\hline
\end{tabular}

Fonte: modificado de Zanden, Taher e Arends (2015). 
Nos Casos 1 e 2, a amostra contém $1 \mathrm{~kg}$ de sal por $\mathrm{m}$ de concreto, o que implica 12,5 $\mathrm{kg}$ de sal por $\mathrm{m}$ de água. O único fluxo de entrada na amostra é de água, ou seja, não há névoa salina, porém no Caso 2 a saída é de água e sal. Nos Casos 3 e 4, inicialmente não há sal na amostra, mas ambas são atingidas por uma névoa de íons $\mathrm{Cl}^{-}$de $19 \mathrm{~kg} / \mathrm{m}$, causando a entrada do sal juntamente com a água. No Caso 3 não há saída do sal, enquanto no Caso 4há uma livre circulação, ou seja, o sal pode entrar e sair da amostra livremente.

A Figura 4 mostra uma boa similaridade nos resultados de cada simulação, obtida para o $3^{\circ}$ mês do ano, em comparação com os de Zanden, Taher e Arends (2015).

Considerando que o terceiro mês é o mais úmido, percebe-se que nos Casos 1 e 2a concentração de sal na amostra permanece constante em $1 \mathrm{~kg} / \mathrm{m}$. Isso ocorre devido à condição do ambiente, que não apresenta névoa salina, mantendo sua concentração constante apesar do aumento da umidade. Em contrapartida, nos Casos 3 e 4há um aumento da concentração de sal justamente devido à alta umidade e a névoa salina presentes no ambiente. Sua combinação permite a entrada de sal na amostra juntamente com a água causando um aumento da concentração de sal. Nesses casos, os pontos mais internos da parede apresentam uma concentração menor por não estarem em contato direto com o sal, o que retarda o aumento da concentração.

A Figura 5 mostra os diferentes resultados dos casos simulados para o $6^{\circ}$ mês, período de umidade intermediária.

Figura 4 - Avanço do cloreto ao longo da amostra para o $3^{\circ}$ mês

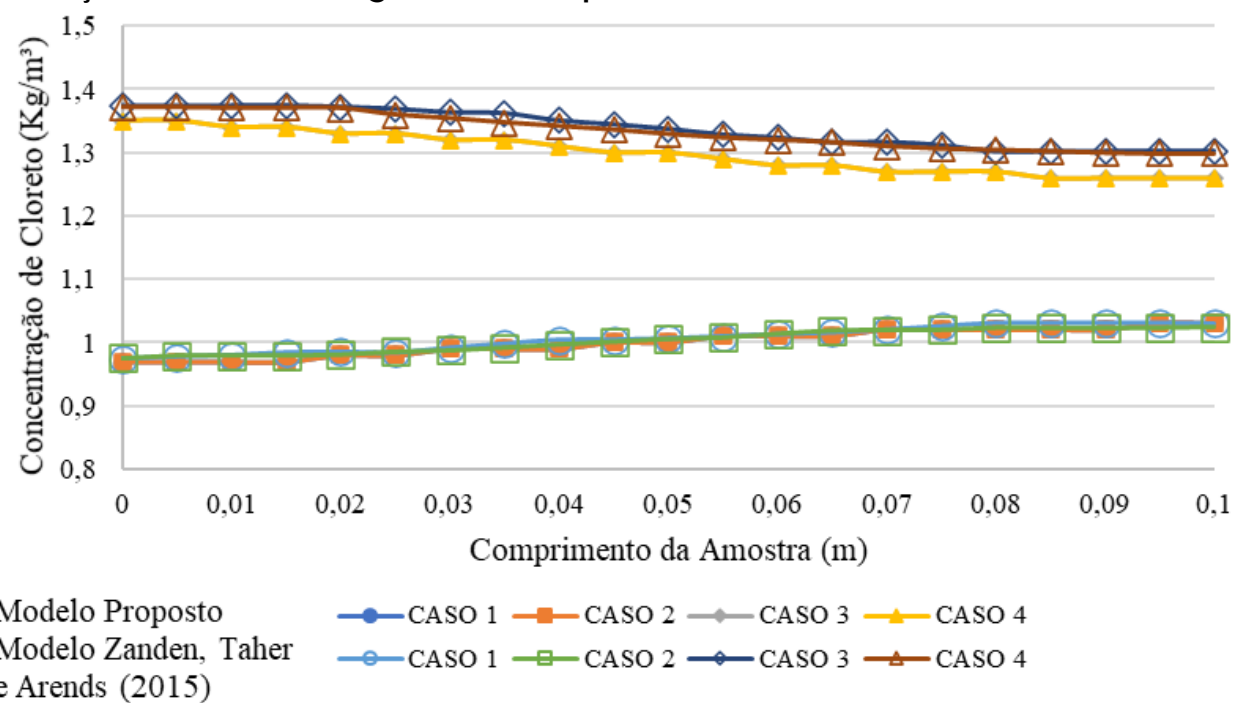

Figura 5 - Avanço do cloreto ao longo da amostra para $6^{\circ}$ mês

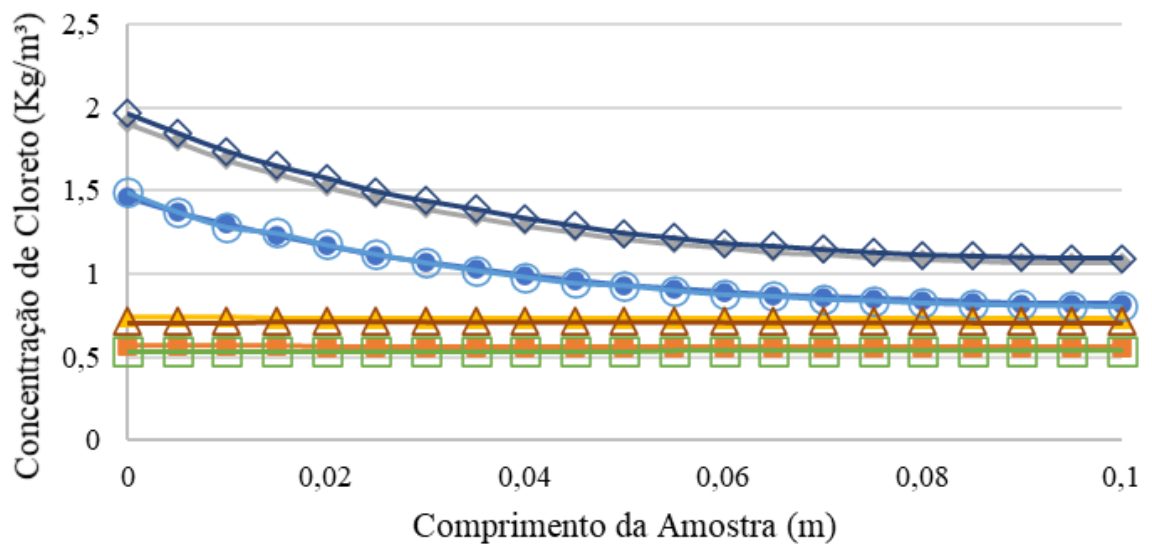

Modelo Proposto

Modelo Zanden, Taher

e Arends (2015)

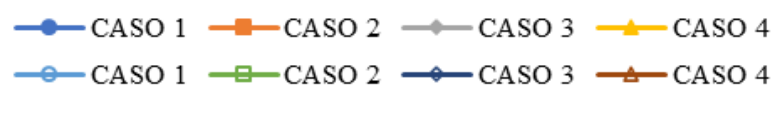


Observa-se uma similaridade entre o comportamento dos Casos 1 e 3 e entre os Casos 2 e 4 . Atribui-se esse fato pela mesma restrição imposta, onde há saída apenas de água nos Casos 1 e 3 e de água e sal nos Casos 2 e 4. A diminuição da umidade no ambiente em relação aos meses anteriores inicia um processo de secagem da amostra. Nesse caso, ocorre a saída de água e sal dissolvido na amostra como um todo, porém, nos Casos 1 e 3, esse transporte não pode ocorrer, causando um aumento da concentração de sal próximo da extremidade. Por outro lado, nos Casos 2 e 4, o sal se desloca até a extremidade e sai da amostra junto com a água, ocasionando a queda da concentração de sal em relação ao $3^{\circ}$ mês, conforme observado na Figura 5.

O $9^{\circ}$ mês se caracteriza por ser o período mais seco, conforme observado na Figura 2. Os resultados para esse caso podem ser observados na Figura 6.

Para analisar a Figura 6, ressalta-se que o Caso 4 apresenta a situação de livre fluxo, onde tanto a água quanto o sal podem entrar e sair da amostra livremente de acordo com as condições do ambiente. Nesse processo, observa-se que, apesar de haver uma grande saída de sal no $9^{\circ}$ mês devido à estiagem, ainda há presença de sal na amostra, mesmo que em pequena quantidade. Isso pode causar o acúmulo de sal na amostra com o tempo, que, por sua vez, pode cristalizar-se com a secagem, levando ao aumento da pressão interna e à consequente formação de fissuras e rachaduras.

Após um ano, a Figura 7 apresenta uma grande diferença na concentração de íons $\mathrm{Cl}^{-}$. No Caso 1 o fluxo é apenas de água, o que causa apenas um movimento interno do sal em seu interior. Quando aumenta a umidade no ambiente, a água tende a entrar na amostra levando o sal em direção da extremidade oposta. Por outro lado, quando a umidade diminui, a água que sai da amostra carrega os íons até os primeiros nós. No Caso 20 comportamento é diferente devido à possibilidade da saída de sal, o que reduz sua concentração a quase zero nos períodos de baixa umidade, mantendo-se constante nos períodos de alta umidade devido à entrada de apenas água na amostra. No Caso 3, apesar do seu comportamento ser muito semelhante ao do Caso 1, haverá um constante aumento da concentração de sal. Isso ocorre porque, ao entrar na amostra com a água, o sal fica preso e não sai nos períodos de secagem da amostra. O Caso 4 apresenta a situação de livre fluxo, em que a água e o sal podem entrar e sair de acordo com as condições do ambiente, aumentando e diminuindo sua concentração em períodos de maior e menor umidade, respectivamente.

De acordo com a comparação realizada entre os estudos de casos propostos por Zanden, Taher e Arends (2015), há uma boa similaridade entre os resultados. As pequenas diferenças podem ser atribuídas à quantidade de nós utilizada, à incerteza da quantidade de dias considerada para cada mês (30 ou 31) e aos diferentes métodos numéricos empregados em cada trabalho.

\section{Figura 6 - Avanço do cloreto ao longo da amostra para o $9^{\circ}$ mês}

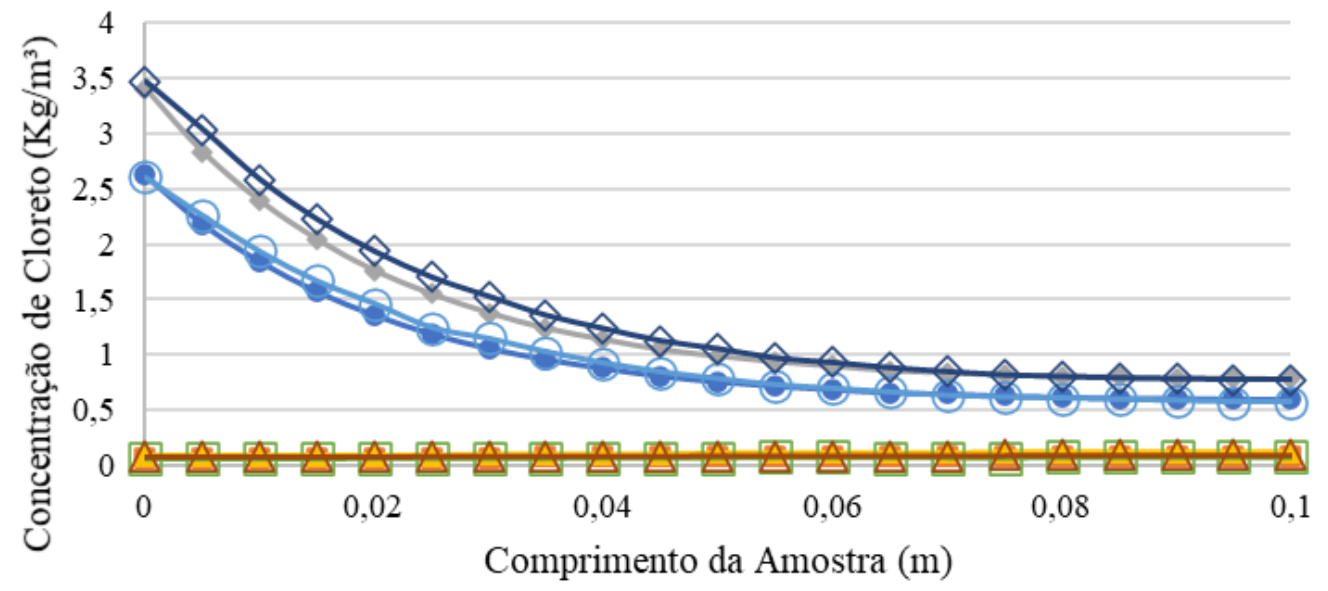

\footnotetext{
Modelo Proposto $\longrightarrow$ CASO $1-$ CASO $2 \multimap$ CASO $3-$ CASO 4

Modelo Zanden, Taher $\multimap$ CASO $1 \multimap$ CASO $2 \multimap$ CASO $3 \longrightarrow$ CASO 4

e Arends (2015)
}

152 Ribas, R. M. M.; Santos, G. H. dos; Okita, V. 
Figura 7 - Avanço do cloreto ao longo da amostra para o $12^{\circ}$ mês
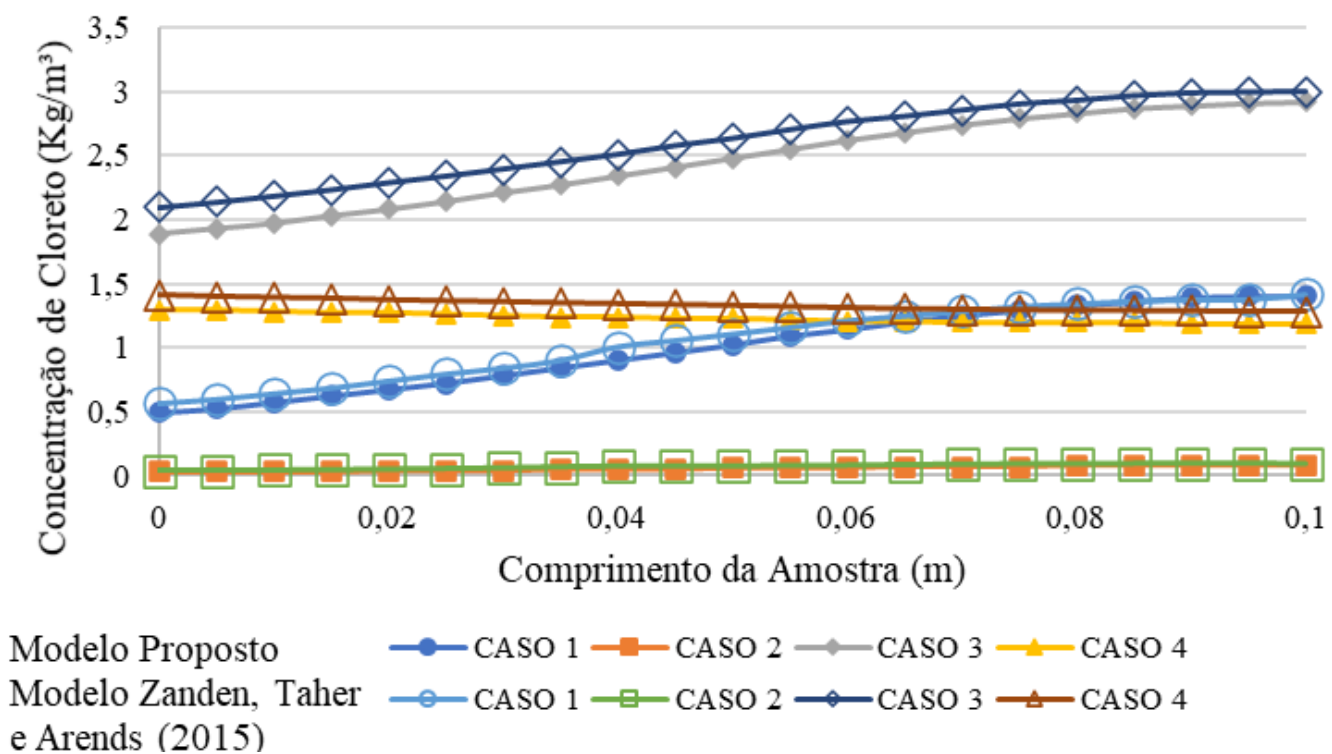

A partir dos resultados obtidos, percebe-se que o principal mecanismo para o transporte de sal é o transporte de umidade. Em períodos em que ocorre uma queda da umidade ambiente ( $6^{\circ}$ e $9^{\circ}$ mês), a amostra seca, e o sal dissolvido é levado para fora (Casos 2 e 4) ou fica preso nos primeiros nós da amostra (Casos 1 e 3). A partir do Caso 4, percebe-se claramente que o fluxo de umidade apresentado no modelo matemático causa mais impacto para o transporte de sal que o gradiente de concentração, isso porque, mesmo com a concentração do ambiente sendo mais alta do que a da amostra, o sal tende a sair devido ao fluxo de água.

A Figura 8 apresenta a simulação de uma situação crítica real, onde é avaliada a concentração de sal (em $\mathrm{kg}$ $\mathrm{Cl}^{-} / \mathrm{m} \mathrm{H}_{2} \mathrm{O}$ ) ao longo da parede de concreto, para o $9^{\circ}$ mês, que é o mês mais seco. Essa análise foi realizada com o objetivo de avaliar a possibilidade de cristalização ao longo do tempo, considerando que durante a secagem os íons $\mathrm{Cl}^{-}$permaneceram no concreto, ou seja, que não houve fluxo de sal saindo da amostra (Caso 3). Escolheu-se esse caso, pois, durante o processo de secagem da amostra, ocorreu a vaporização da água próxima à superfície esquerda, concentrando o sal nessa região. A perda de água pelos íons $\mathrm{Cl}^{-}$facilita a nucleação do sal, aumentando com o tempo sua cristalização e podendo causar desde um acréscimo da pressão interna até o rompimento do concreto.

Como mostra a Figura 8, a concentração de cloreto é maior na extremidade mais externa da parede e mais reduzida nas regiões mais internas. Observando o limite de solubilidade do cloreto de sódio a $350 \mathrm{~kg} / \mathrm{m} \mathrm{para}$ $20^{\circ} \mathrm{C}$ (representado pela linha pontilhada), nota-se que no $9^{\circ}$ mês ainda não há possibilidade de formação de cristais. Porém, a concentração do sal chega muito próximo do limite de solubilidade, o que evidencia a grande possibilidade de cristalização a partir do primeiro ano. Verifica-se também que a concentração do sal nos pontos mais internos tende a aumentar, podendo atingir esse limite com o decorrer do tempo.

\section{Conclusão}

O presente estudo buscou analisar o comportamento do transporte de sal em estruturas de concreto. Para isso, inicialmente se apresentou o modelo matemático para o transporte de calor, umidade e sal em um meio poroso. As equações governantes foram discretizadas pelo método dos volumes finitos gerando um sistema algébrico, que foi solucionado por meio do algoritmo MTDMA, implementado em $\mathrm{C}++$. O método de resolução adotado permite solucionar as equações de forma simultânea para cada nó do sistema, assegurando uma boa robustez numérica. 
Figura 8 - Concentração de cloreto na amostra para o período de 9 meses

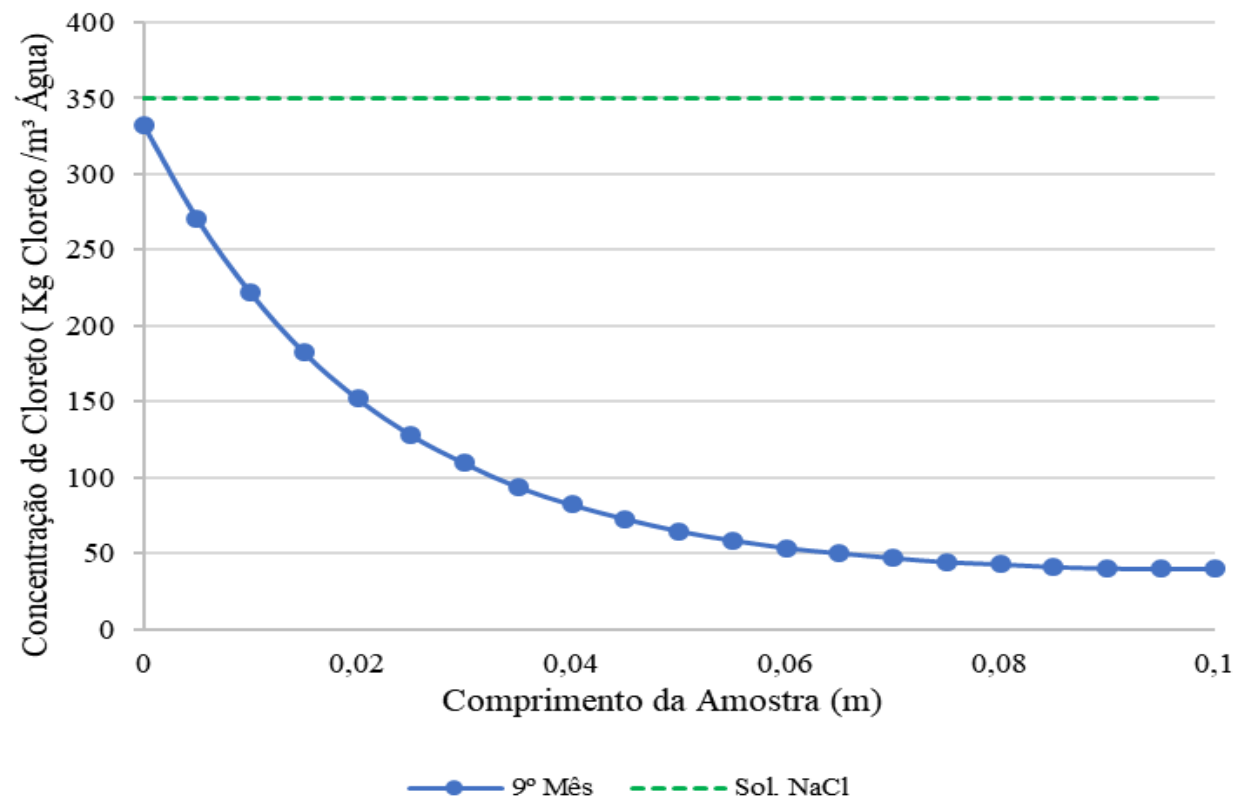

Para verificar o modelo proposto, realizou-se a comparação com o modelo de Zanden, Taher e Arends (2015), com o qual apresentou satisfatória similaridade apesar de suas diferenças de modelagem e discretização. Zanden, Taher e Arends (2015) utilizaram o método de Crank-Nicolson para discretizar as equações e o implementaram no software MATLAB. Sabe-se que esta forma de solução pode requerer um grande tempo computacional, levando em consideração o tamanho do domínio (problemas multidimensionais) e o período de simulação. O modelo proposto foi discretizado pelo método dos volumes finitos, em que foi utilizado o algoritmo MTDMA para a solução acoplada do sistema, garantindo uma boa robustez numérica. $\mathrm{O}$ modelo proposto também inclui a equação da energia, que permite a simulação de problemas não isotérmicos, a qual será explorada nos próximos trabalhos. A partir dos resultados apresentados, pode-se perceber a grande influência da umidade no transporte de sal durante os processos de imbibição e secagem. Os íons $\mathrm{Cl}^{-}$, ao entrarem em contato com a água no interior da amostra, ficam em solução e são levados de acordo com a sua movimentação. Em processos de imbibição, a umidade do ambiente adentra a amostra e alcança até os últimos nós, carregando consigo o sal. No caso do processo de secagem, a amostra perde umidade para o ambiente, que está mais seco. Nos processos em que não há a presença de gradiente de umidade entre a amostra e o ambiente, a movimentação do sal no interior da amostra ocorre apenas por conta do gradiente de concentração. Nesse caso, o processo é mais lento e ocorre na direção do equilíbrio em todos os pontos da amostra.

A boa similaridade entre os resultados dos casos apresentados na literatura mostra que o modelo apresentado tem potencial para analisar outras condições de contorno, como a análise do transporte de sal afetado por gradientes de temperatura. $\mathrm{O}$ modelo proposto também pode ser incrementado considerando o efeito da cristalização nas equações governantes, permitido avaliar a possibilidade de sua formação e ainda prever e acompanhar sua nucleação, crescimento e dissolução ao longo do tempo. Apesar das simplificações impostas no modelo, o processo e o tempo para o início da recristalização podem ser previstos, obtendo-se informações importantes para prevenir a deterioração em estruturas de concreto.

Algumas simplificações adotadas no modelo poderão ser verificadas em trabalhos futuros, como o efeito da cristalização nos poros e consequentemente no transporte, a influência da concentração do sal no transporte da umidade, as interações do sal com a parede do poro e os efeitos da carbonatação no concreto. Neste trabalho foram consideradas também condições extremas na superfície do concreto (todo ou nenhum sal entrando com fluxo de água). Outras condições de contorno mais realísticas poderão ser modeladas, levando em consideração o efeito da temperatura, que certamente desempenha um papel importante neste processo.

154 Ribas, R. M. M.; Santos, G. H. dos; Okita, V. 


\section{Referências}

ABABNEH, A.; BENBOUDJEMA, F.; XI, Y. Chloride penetration in nonsaturated concrete. Journal of Materials in Civil Engineering, v. 15, p. 183-191, 2003.

ARENDS, T. et al. A model describing water and salt migration in concrete during wetting/drying cycles. In: INTERNATIONAL CONFERENCE ON NON-TRADITIONAL CEMENT AND CONCRET, 5., Brno, 2014. Proceedings [...] Brno, 2014.

BENITE, A. Emissões de carbono e a construção civil. Centro de Tecnologia de Edificações. São Paulo, 2011. Disponível em: http://www.cte.com.br/imprensa/2011-02-27-emissoes-de-carbono-e-a-construcaociv/. Acesso em: 29 mar. 2017.

DELLINGHAUSEN, L. M. et al. Retração total e penetração de cloretos em concretos com cimento Portland branco e escória de alto forno. Ambiente Construído, Porto Alegre, v. 9, n. 4, p. 45-35, out./dez. 2009.

DERLUYN, H.; MOONEN, P; CARMELIET, J. Modelling of Moisture and Salt Transport Incorporating Salt Crystallization in Porus Media. Journal of Mechanics and Physics of Solids, v. 63, p. 242-255, 2014.

GORNINSKI, J. P.; KAZMIERCZAK, C.S. Avaliação da resistência química de concretos poliméricos em ambientes agressivos. Ambiente Construído, Porto Alegre, v. 8, n. 1, p. 105-113, jan./mar. 2008.

HELENE, P. R. L. Introdução a durabilidade no projeto das estruturas de concreto. Ambiente Construído, Porto Alegre, v. 1, n. 2, p. 45-57, jul./dez. 1997.

KROPP, J. Chlorides in concrete. In: KROPP, J.; HILSDORF, K. (ed.). Performance criteria for concrete durability. Boca Ratón: CRC Press, 1995. RILEM Report 12.

LI, C.; LI, K.; CHEN, Z. Analysis of moisture influential depth in concrete during drying-wetting cycles. Tsinghua Science and Technology, v. 13, n. 5, p. 696-701, 2008.

MARTINS, A. A. A. Fenómenos de transporte em meios porosos: escoamento monofásico e transporte de massa. Porto, 2006. Dissertação (Doutorado em Engenharia Química) - Faculdade de Engenharia, Universidade do Porto, Porto, 2006.

MEDEIROS, M. H. F.; ANDRADE, J. J.O.; HELENE, P. Durabilidade e vida útil das estruturas de concreto. In: ISAIA, G. C. Concreto: Ciência e Tecnologia. São Paulo: IBRACON, 2011.

MEHTA, P. K.; MONTEIRO, P. J. M. Concreto: estrutura, propriedades e materiais. São Paulo: PINI, 2008.

MENDES, N.; PHILIPPI, P. C.; LAMBERTS, R. A new mathematical method to solve highly coupled equations of heat and mass transfer in porous media. International Journal of Heat and Mass Transfer, $\mathrm{V}$. 45, n. 3, p. 509-518, 2002.

PATANKAR, S. V. Numerical heat transfer and fluid flow. New York: Hemisphere ,1980. (Series in computational methods in mechanics and thermal sciences).

PEDROSO, F. L. Concreto: as origens e a evolução do material construtivo mais usado pelo homem. Concreto \& Construções, São Paulo, v. 37, n. 53, 2009.

RIZZON, A. C.; ANDRADE, J. J. O. Vida útil de concretos brancos quando inseridos em ambiente com cloretos :a influência do cimento e do coeficiente de difusão. Ambiente Construído, Porto Alegre, v. 7, n. 3, p. 21-34, jul./set. 2007.

ROCHA, J. H. A.; PÓVOAS, Y. V. Deteç̧ão de corrosão em concreto armado com termografia infravermelha e ultrassom. Ambiente Construído, Porto Alegre, v. 19, n. 3, p. 53-68, jul./set. 2019.

SANTOS, G. H.; MENDES, N. Combined Heat, Air and Moisture (HAM) transfer model for porous building materials. Journal of Building Physics, v. 32, p. 203-220, 2009.

SUN, C. et al. Numerical and experimental study of moisture and chloride transport in unsaturated concrete. Elsevier Journal, v. 189, p. 1067-1075, 2018.

ZANDEN, A. J. J van der; TAHER, A.; ARENDS, T. Modelling of water and chloride transport in concrete during yearly wetting/drying cycles. Elsevier Journal, v. 81, p. 120-129, 2015. 


\section{Agradecimentos}

Os autores agradecem ao Conselho Nacional de Desenvolvimento Científico e Tecnológico (CNPq), à Coordenação de Aperfeiçoamento de Pessoal de Nível Superior (CAPES) e à Fundação Araucária pelo suporte financeiro.

Rhayssa Maryell Marra Ribas

Programa de Pós-Graduação em Engenharia Mecânica | Universidade Tecnológica Federal do Paraná | Rua Doutor Washington Subtil Chueire, 330, Jardim Carvalho | Ponta Grossa - PR - Brasil | CEP 84017-220 | E-mail: rhayssa@alunos.utfpr.edu.br

Gerson Henrique dos Santos

Programa de Pós-Graduação em Engenharia Mecânica | Universidade Tecnológica Federal do | Paraná | E-mail: gsantos@utfpr.edu.br

Viviane Okita

Programa de Pós-Graduação em Engenharia Mecânica | Universidade Tecnológica Federal do Paraná | E-mail: okitaviviane@gmail.com

\section{Ambiente Construído}

Revista da Associação Nacional de Tecnologia do Ambiente Construído

Av. Osvaldo Aranha, $99-3^{\circ}$ andar, Centro

Porto Alegre - RS - Brasil

CEP 90035-190

Telefone: +55 (51) 3308-4084

www.seer.ufrgs.br/ambienteconstruido www.scielo.br/ac

E-mail: ambienteconstruido@ufrgs.br 